\title{
Determination of Cutoff Point of an Early Energy Target to Maximize Better Clinical Outcomes in SCU Patients with Cerebral Hemorrhage
}

\author{
Noriko Kurokawa ${ }^{1,2}$, Yoko Hokotachi ${ }^{1,3}$ and Teruyoshi Amagai ${ }^{4 *}$ \\ ${ }^{1}$ Administration Food Science and Nutrtion Major, Graduate School of Human Environmental Sciences, Mukogawa Women's Univeristy, \\ Nishinomiya,Japan \\ ${ }^{2}$ Department of Clinical Nutriton, Kitano Hospital, The Tazuke Kofukai Medical Reserch Institute, Osaka, Japan \\ ${ }^{3}$ Department of Clinical Nutriton, Takarazuka Dai-ichi Hospital,Takarazuka, Japan \\ ${ }^{4}$ Administration Food Science and Nutrtion, School of Human Environmental Sciences, Mukogawa Women's Univeristy, \\ Nishinomiya,Japan \\ *Corresponding author: Teruyoshi Amagai, Department of Food Sciences and Nutrition, School of Environmental Sciences, \\ Ikebiraki-Cho, Nishinomiya, 663-8558, Japan
}

\begin{abstract}
ARTICLE INFO
Received: 幽 October 10, 2019

Published: 幽 October 21, 2019

Citation: Noriko Kurokawa, Yoko Hokotachi, Teruyoshi Amagai. Determination of Cutoff Point of an Early Energy Target to Maximize Better Clinical Outcomes in SCU Patients with Cerebral Hemorrhage. Biomed J Sci \& Tech Res 22(2)-2019. BJSTR. MS.ID.003716.
\end{abstract}

Keywords: Stroke; Cerebral Hemorrhage; Energy Target; Cutoff Point; Outcome

\section{ABSTRACT}

Aim: To examine working hypothesis that patients with cerebral hemorrhage $(\mathrm{CH})$ have worse outcomes compared with cerebral infarction (CI) and examine that the cutoff point of \% energy intake (EI) exists to maximize better outcomes in $\mathrm{CH}$ patients.

Methods: All consecutive acute stroke patients with cerebral hemorrhage $(\mathrm{CH})$, or cerebral infarction (CI), admitted to a single institution, were enrolled. To discriminate $\mathrm{CH}$ patients with poor outcome, the cutoff point of \% energy intake (EI) was examined.

Results: Three hundred and sixty patients were enrolled and a hundred and thirty were excluded. Two hundred and thirty patients were analyzed. Among $\mathrm{CH}$ patients, the cutoff point of \%EI was determined at $75 \%$ to maximize better clinical outcomes.

\section{Conclusion}

a) $\mathrm{CH}$ patients have worse outcomes compared with $\mathrm{CI}$ patients.

b) A cutoff point of $\% \mathrm{EI}$ was determined at $75 \%$ of the target to maximize better clinical outcomes in $\mathrm{CH}$ patients.

\section{Introduction}

\section{Background}

Stroke is the third leading cause of death [1]. Among stroke subtypes, cerebral ischemia (CI), cerebral hemorrhage $(\mathrm{CH})$ have directly insult to cerebral neurons, whereas subarachnoid hemorrhage does not. Comparing outcome of $\mathrm{CH}$ with $\mathrm{CI}$, patients with $\mathrm{CH}$ have been reported to have higher in-hospital mortality [Odds ratio $=2.9$ ] [2]. Under these circumstances, we hypothesized that $\mathrm{CH}$ patients have poorer outcomes compared with patients with $\mathrm{CI}$ and that an energy management set at $75 \%$ of target was associated with better outcome. Here, target of energy intake (TEI) was set at $25 \mathrm{kcal} / \mathrm{kg}$ of actual body weight / day according to the statement of guidelines provided by American Society for Parenter al and Enteral Nutrition [3], and European Society for Parenteral and Enteral Nutrition [4]. Then if our hypothesis was correct, then examine that the cutoff point of TEI exists to maximize better outcomes. To test our hypotheses, all data was collected and analyzed in patients with $\mathrm{CI}$ and $\mathrm{CH}$ admitted to stroke care unit (SCU) in a single tertiary institute.

\section{Methods}

All consecutive acute stroke patients, admitted to a single institution between January and June 2015, were enrolled as subjects of the study. They were divided into two groups, diagnosed with $\mathrm{CH}$ or $\mathrm{CI}$ as primary diagnosis. The exclusion criteria were: 
(1) The length of stay in hospital $<7$ days because the shorter stay could not enough to test an effect of nutritional support on a clinical outcome,

(2) Primary diagnosis was not $\mathrm{CH}$ or $\mathrm{CI}$,

(3) Primary diagnosis was subarachnoid hemorrhage because it does not seem to influence focal neurological region,

(4) Missing data of height or weight.

The collected data of an individual subject were including in the following five categorized parameters:

(1) Demographics, including age, sex, body weight, body mass index (BMI, expressed in unit of $\mathrm{kg} / \mathrm{m}^{2}$ ), Charlson comorbidity index (CCI) to evaluate individual comorbidity, stroke subtype (CI or $\mathrm{CH}$ ), National Institutes of Health Stroke Scale (NIHSS) for estimation of neurological deficits. All was evaluated at admission,
(2) Blood test parameter of C-reactive protein (CRP) measured during staying in SCU,

(3) Nutritional parameters, including daily energy intake (EI: kcal / kg of actual body weight / day) for the first seven days staying in SCU. Here, when subject has discharged within six days from SCU, an average daily EI was calculated until the day before discharge from SCU. In addition, nutritional route, including oral or the others (enteral or parenteral) was also classified,

(4) Outcome parameters, including the length of stay in SCU as the primary outcome, and the highest CRP during stay in SCU, the presence of CRP $\geq 6.0 \mathrm{mg} / \mathrm{dL}$, both as the second outcomes.

Then, the following analyses were conducted in analysis 1 , and 2 (Figure 1). Given the nature of this study, the requirement for informed patient consent was considered unnecessary.

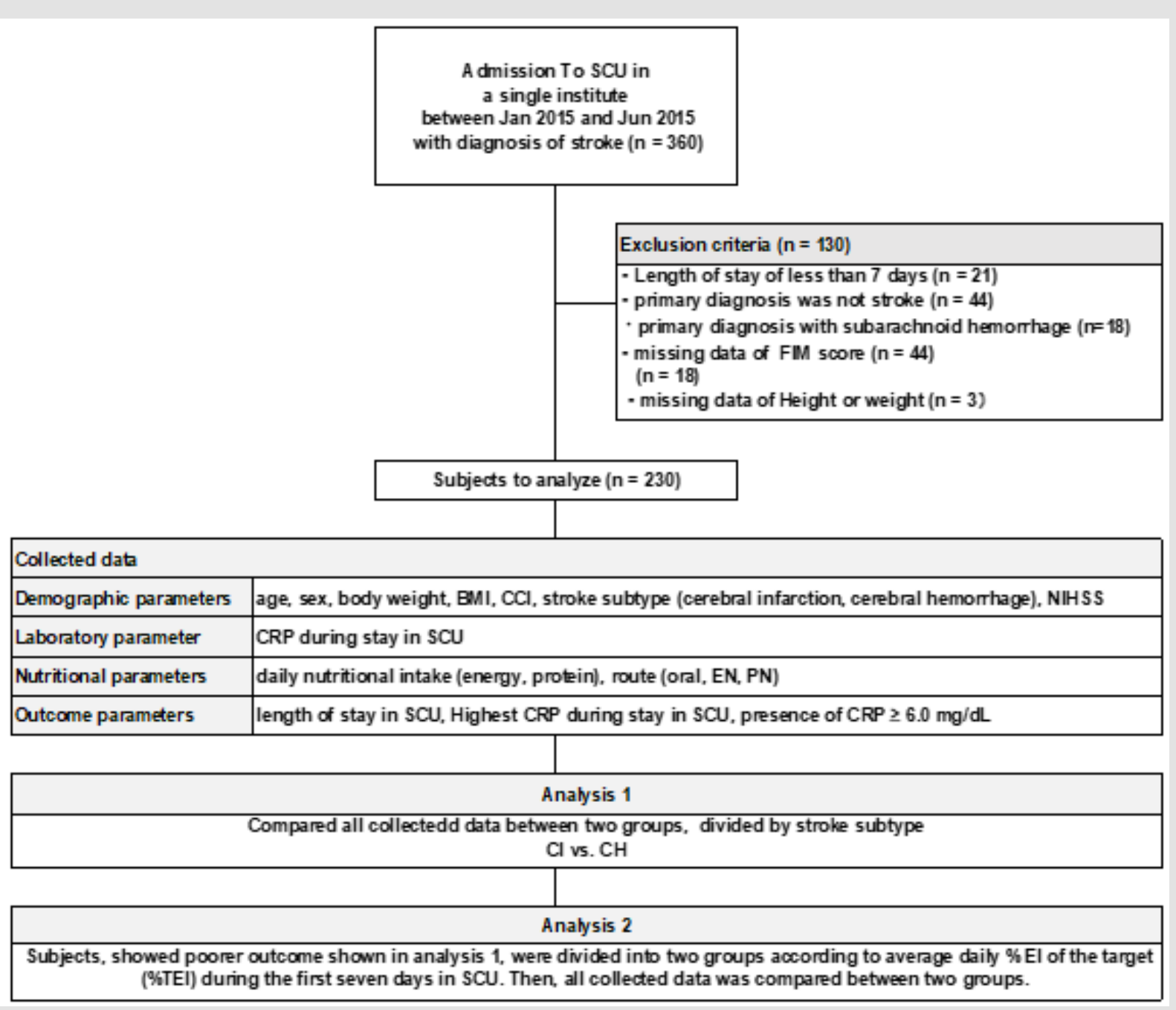

Figure 1: The flowchart of this study.

Abbreviations. BMI: body mass index, CCI: Charlson comorbidity index, CH: cerebral hemorrhagem, CI; cerebral infarction, CRP: C-reactive protein, EI: energy intake, EN: enteral nutritoin, FIM: functional independence measure, NIHSS: National Institutes of Health Stroke Scale, PN: parenteral nutrition, SCU: stroke care unit, TEI: energy intake of the target. 


\section{Analysis 1}

To examine our hypothesis that $\mathrm{CH}$ patients have worse outcomes compared with that of CI patients, all included subjects was divided into two groups according to their primary stroke subtype, $\mathrm{CI}$ or $\mathrm{CH}$. Then, all collected data was compared between two groups to know which group show significantly poorer outcome. Here, group that showed poorer outcome were proceeded to analysis 2 .

\section{Analysis 2}

To examine our hypothesis that patients fed with \%EI of the target is associated with maximizing clinical outcome in SCU, all $\mathrm{CH}$ subjects were divided into two subgroups by EI of \% target energy (TEI), < vs. $\geq 75 \%$, and followed by added the same analyses with the TEI set at $50 \%$ and $25 \%$. Then, comparisons of all collected data was conducted between two subgroups to which target \%EI, 75\%, $50 \%$, or $25 \%$ of $\%$ of target EI, is strongest to show significance in outcomes.

\section{Statistical Analysis}

The outcome parameters in the two groups were divided into different categories and compared using the Mann-Whitney U test for continuous variables and the chi-square test or Fisher's exact test for categorical variables. All analyses were performed using SPSS Statistics version 24 (IBM Corp., Armonk, NY, USA), and significance was examined at $\mathrm{P}<0.05$.

\section{Results}

Three hundred and sixty patients were enrolled and a hundred and thirty were excluded. Two hundred and thirty patients were included to analyze in further analyses (Figure 1).

\section{Result 1-Results for analysis1}

Compared with patients among all patients with $\mathrm{CH}$ and $\mathrm{CI}$, CH patients showed higher NIHSS (10vs.3, p < 0.001), and their outcome parameters showed that longer length of stay in SCU (7 vs. $5, \mathrm{p}<0.001)$ and higher CRP during stay in SCU significantly (1.6 vs. $0.2, \mathrm{p}<0.001$ ) (Table 1).

Table 1: Demographic and anthropometric characteristics, in the cerebral infarction and cerebral hemorrhage group.

\begin{tabular}{|c|c|c|c|c|}
\hline & Total $(n=230)$ & Cerebral Infraction $(n=165)$ & Cerebral Hemorrhage $(n=65)$ & P Value \\
\hline \multicolumn{5}{|c|}{ Demographic Parameters } \\
\hline Age, Years & $71(63,78)$ & $71(64,78)$ & $87(57,79)$ & 0.083 \\
\hline Males, n (\%) & $146(63)$ & $100(61)$ & $46(71)$ & 0.172 \\
\hline Height, $\mathrm{cm}$ & $163(153,169)$ & $161(153,167)$ & $165(157,170)$ & 0.021 \\
\hline Weight, kg & $60(51,68)$ & $60(50,66)$ & $62(55,72)$ & 0.040 \\
\hline BMI, kg/m² & $23(21,25)$ & $23(21,25)$ & $23(21,26)$ & 0.549 \\
\hline NIHSS Score & $4(1,10)$ & $3(1,7)$ & $10(5,17)$ & $<0.001$ \\
\hline CCI & $2(1,4)$ & $2(1,4)$ & $3(1,4)$ & 0.260 \\
\hline Old Cerebral Infraction, n (\%) & $64(28)$ & $46(28)$ & $18(28)$ & 1.000 \\
\hline \multicolumn{5}{|c|}{ Nutritional Parameters } \\
\hline $\begin{array}{l}\% \text { Energy intake of the target during the } \\
\text { first s even days in SCU (\%) }\end{array}$ & $80(54,97)$ & $88(70,102)$ & $53(24,79)$ & $<0.001$ \\
\hline \multicolumn{5}{|c|}{ Outcome Parameters } \\
\hline Length of stay in SCU & $6(4,8)$ & $5(3,8)$ & $7(5,11)$ & $<0.001$ \\
\hline Highest CRP during stay in SCU, mg/dl & $0.4(0.1,2.6)$ & $0.2(0.1,1.5)$ & $1.6(0.5,4.4)$ & $<0.001$ \\
\hline $\mathrm{CRP} \geq 6.0 \mathrm{mg} / \mathrm{dl}, \mathrm{n}(\%)$ & $26(11)$ & $12(7)$ & $14(22)$ & 0.039 \\
\hline
\end{tabular}

Note: Data are expressed in medium (25\% tile, $75 \%$ tile).

Cerebral infarction vs. Cerebral hemorrhage: Mann - Whitney's U test and the chi - square or Fisher's exact test for categorical variables

Abbreviations: BMI: Body mass index, CCI: Charlson comorbidity index. CRP: C-reactive protein, FIM : Functional Independence Measure, NIHSS: National Institutes of Health Stroke Scale, SCU: stroke care unit.

\section{Result 2-Results for Analysis 2}

As $\mathrm{CH}$ patients showed significantly severe and poor outcomes, $\mathrm{CH}$ patients were proceeded to analysis 2 . This analysis showed that the cutoff point of $\%$ EI was determined at $75 \%$ to be associated with better clinical outcomes, such as significantly shorter length of stay (LOS) in SCU (Table 2). Additionally, the same differences were observed also analyses of $50 \%$ and $25 \%$ studies. From these observations, the upper limit to maximize better outcomes must 
be determined at $75 \%$. In other words, $\mathrm{CH}$ patients fed with $\% \mathrm{EI} \geq$ $75 \%$ of target seemed to maximize better clinical outcomes.

\section{Discussion}

An Impact of an Early $75 \%$ of the Target Energy Intake is Associated with Maximizing Clinical Outcomes in Critically Ill Stroke Patients

In the present study, the length of stay in SCU and CRP values were applied as outcome parameters. Among them, CRP $\geq 6.0 \mathrm{mg} /$ $\mathrm{dL}$ was considered as a biomarker of infectious events in the older patients [5]. In analysis 1, as $\mathrm{CH}$ patients showed poor outcome compared with patients with $\mathrm{CI}$, next question was raised whether an energy management have an impact on clinical outcome or not. As the results showed in analysis 2, $\mathrm{CH}$ patients managed with smaller energy $\geq 75 \%$ of TEI seems associated with significantly lower frequent of CRP $<6.0 \mathrm{mg} / \mathrm{dL}$ compared with patients fed with larger energy $\geq 75 \%$ of the target. This result could be interpreted that more frequent lower CRP in SCU and shorten LOS in SCU could be achieved by $\% \mathrm{EI} \geq 75 \%$ of the target. In these contexts, the cutoff point of $\%$ EI might exist at $75 \%$.

Table 2: Comparison of Clinical outcomes in two groups in the cerebral Hemorrhage: <\%TEI vs. \% TEI $\leq \%$ TEI groups defined by their \% energy intake of the target. Here, energy target was set at 25kcal $/ \mathrm{kg}$ of actual body weight, according to E SPEN guideline

\begin{tabular}{|c|c|c|c|c|c|c|c|c|c|}
\hline Cutoff \% Energy of the target & \multicolumn{3}{|c|}{$\mathbf{2 5 \%}$} & \multicolumn{3}{|c|}{$\mathbf{5 0} \%$} & \multicolumn{3}{c|}{$75 \%$} \\
\hline Sub-group & $<\mathbf{2 5 \%}$ & $\mathbf{2 5 \%} \leq$ & P Value & $<\mathbf{5 0 \%}$ & $\mathbf{5 0 \%} \leq$ & P Value & $<75 \%$ & $\mathbf{7 5 \%} \leq$ & P Value \\
\hline Subjects number & $\mathbf{1 6}$ & $\mathbf{4 9}$ & & $\mathbf{3 1}$ & $\mathbf{3 4}$ & & $\mathbf{4 6}$ & $\mathbf{1 9}$ & \\
\hline LOS in SCU, DAYS & $8(5,11)$ & $7(5,10)$ & 0.748 & $8(5,12)$ & $7(5,9)$ & 0.081 & $8(5,11)$ & $6(4,8)$ & 0.021 \\
\hline Highest CRP, mg/dl & $\begin{array}{c}5.0(1.9, \\
19.6)\end{array}$ & $\begin{array}{c}0.9(0.3, \\
2.7)\end{array}$ & $<0.001$ & $16(1.7,7.9)$ & $0.7(0.2,1.8)$ & $<0.001$ & $9(5,11)$ & $0.3(0.2$, & $<0.001$ \\
\hline $\begin{array}{c}\text { Presence of CRP 26.0 mg/di } \\
\text { during stay in SCU, n (\%) }\end{array}$ & $7(44)$ & $5(10)$ & 0.006 & $10(32)$ & $2(6)$ & 0.009 & $10(5,11)$ & $1(5)$ & 0.073 \\
\hline
\end{tabular}

Note: Data are expressed in medium (25\% tile, $75 \%$ tile).

Abbreviations CRP: C-reactive protein, E-L: Energy intake < of target, E-H: Energy intake $\geq$ of target, SCU : stroke care unit.

The Reason Why 75\% of \% TEI is Cutoff Point to Maximize Better Clinical Outcomes in Stroke Patients

To consider how much energy intake must be associated with outcomes, we would discuss three scenarios, such as \%EI set at $75 \%,>100 \%$, and meta-analysis, separately.

\%EI $\geq 75 \%$ of the Target: As A.S.P.E.N. [6] and ESPEN [7] guidelines has similarly recommended to enhance an early enteral nutrition to achieve better outcome for critically ill patients, addressing attentions to the question how much energy is associated with an improvement of outcomes in critically ill settings, a study entitled the tight calorie control study (TICACOS) seems to be informative. The authors reported that critically ill patients were divided into two groups by the method to calculate EI: one was calculated by indirect calorimetry (study group) and another was set by weight-based calculation, $25 \mathrm{kcal} / \mathrm{kg} /$ day as the target (control group). The result, surprisingly contrary to our expectations, showed that the control group, setting at $25 \mathrm{kcal} /$ $\mathrm{kg} /$ day or $76 \%$ of the target, had significantly shorter duration of mechanical ventilation (10.5 vs. 161.1 days, $\mathrm{p}=0.03$ ), fewer infectious complications ( 20 vs. 37, p=0.05), and shorter LOS in ICU (11.7 vs. 17.2 days, $\mathrm{p}=0.04$ ) compared with study group set at $104 \%$ of the target [8].

In the other studies, underfeeding is also reported associated with adverse events, such as hypoglycemia, hypothermia, infectious complications, delayed wound healing, and an impaired immunity $[9,10]$. Similarly to this results, another studies also reported that $<$
$70 \%$ energy provision was associated with significantly higher 30 day mortality compared with $>70 \%$ of the target (31.5 vs. 11.1 $\%, \mathrm{p}=0.01$ ) [11], nutritional guideline also showed that permissive, underfeeding enteral energy provisions have reduced hospital mortality compared with targeted feeding [OR $=0.71,95 \%$ CI. 0.50 $0.99, p=0.04$ ] [12]. The figure of this cutoff point of EI, 75\% is equal to $\%$ [(total energy expenditure (TEE) - physical energy expenditure (PEE) / TEE) [13]. From this result, our observational cutoff point might be equal to energy expenditure without physical activity, such as staying in SCU. Summing up all of results above mentioned could be interpreted that energy management with cutoff strategy set at $75 \%$ of $\%$ EI in an early period in ICU could have an impact on clinical outcomes.

\%EI > $100 \%$ is Associated with Adverse Events: Moving to overfeeding with $>100 \%$ TEI, a cohort study also confirmed that both overfeeding, defined $>110 \%$ and underfeeding, defined $<70 \%$ of the target [8], were reported harmful for critically ill patients [14]. Here, an overfeeding nutritional support has been reported associated with hyperglycemia, hyperlipidemia, and hypercapnia is associated with impairments of autophagy [15]. From these observations, energy management $>100 \%$ of the target seems harmful associated with overfeeding of nutrients mentioned above. However, it must be examined by well-designed studies whether the ceiling of TEI exists or not.

Meta-Analysis of Studies Regarding \%EI of the Target: Cochrane meta-analysis has concluded that there is an uncertainty 
on comparing effects of hypocaloric with standard energy support in length of hospital and SCU stay, infectious complications and the length of mechanical ventilation, because of very low evidence [16]. To extract a conclusive energy management in critically ill settings, although our study showed EI $\geq 75 \%$ of the target was associated with better outcomes, further analyses must be studied to confirm definitive target $\%$ EI.

\section{Prioritizing an Enteral Route for Maintenance of Integral Gut Immunity in Critically Ill Patients}

The nutritional route, parenteral vs. enteral, or oral, must be considered to achieve better outcomes. In general, an enteral nutritional route has been reported to enhance immunity to prevent infectious events [17]. A nutritional management through enteral route decreases infectious complications and LOS in SCU [18]. One of the reasons to explain why enteral route did not show advantage might be that enteral hypo-perfusion might interrupt enteral digestion and absorption of the nutrients. In addition, intestinal alkaline phosphatase (iAP) has been proved to be expressed in intestinal epithelial cells to exclude endotoxin and maintain integral gut immunity $[19,20]$. However, iAP has never been proved its clinical roles I critically ill settings. At the present, roles of iAP regarding an effectiveness of nutritional route must be studied in the further analyses.

\section{Limitations}

First, it is a retrospective study. To draw the definite conclusion of energy intake for $\mathrm{CH}$ patients, prospective, randomized study must be necessary. Second, the cutoff point of \%EI was resulted from a single facility and validity has not yet been fully verified. Third, the sample size was also too small to draw definitive conclusion. The multi-institutional studies are warranted to resolve these limitations.

\section{Conclusion}

This study could draw the following two conclusions:

1) $\mathrm{CH}$ patients have worse outcomes compared with $\mathrm{CI}$ patients.

2) A cutoff point of $\%$ EI was determined at $75 \%$ of the target to maximize better clinical outcomes, such as shorter stay in SCU and lower frequency CRP $\geq 6.0 \mathrm{mg} / \mathrm{dL}$, in $\mathrm{CH}$ patients

\section{Conflict of Interest}

The authors declare that there is no conflict of interest.

\section{Author Contribution}

Conceptualization: Kurokawa N, Hokotachi Y, Amagai T, Project administration: Kurokawa N, Amagai T, Writing - original draft: Kurokawa N. Writing - review and editing: Kurokawa N, Hokotachi Y, Amagai T. Approval of final manuscript: all authors.

\section{Acknowledgement}

The authors appreciate the kind allowance of collection of all data including this study permitted by President of studied institute.

\section{Ethical Board Approval}

The approval for the study was obtained from the ethics committee of the study institution (approval number: 120066).

\section{Funding}

This research received no specific grant from any funding agency in the public, commercial, or not-for-profit sectors.

\section{References}

1. Japanese Ministry of Health. (2017) Labour and Welfare: The statistics of demographic reports.

2. Maestre Moreno JF, Fernández Pérez MD, Triguero Cueva L, Gutiérrez Zúñiga R, Herrera García JD, et al. (2017) Stroke-related mortality in a tertiary care hospital in Andalusia: Analysis and reflections. Neurologia. 32(9): 559-567.

3. Taylor BE, Mc Clave SA, Martindale, RG, Warren MM, Johnson DR, et al. (2016) Society of Critical Care Medicine; American Society of Parenteral and Enteral Nutrition. Guidelines for the provision and assessment of nutrition support therapy in the adult critically ill patient: Society of Critical Care Medicine (SCCM) and American Society for Parenteral and Enteral Nutrition (A.S.P.E.N.). Crit Care Med 44: 390-438.

4. Kreymann KG, Berger MM, Deutz NE, Hiesmayr M, Jolliet P, et al. (2006) ESPEN guidelines on enteral nutrition: intensive care. Clin Nutr 25(2): 210-223.

5. Liu A, Van Nguyen H, Ong B, Shen Q, Kamalasena D. (2010) Serum C-reactive protein as a biomarker for early detection of bacterial infection in the older patients. Age Ageing 39(5): 559-565.

6. Mc Clave SA, Taylor BE, Martindale RG, Warren MM, Johnson DR, et al. (2016) Society of Critical Care Medicine; American Society for Parenteral and EnteralNutrition. Guidelines for the Provision and Assessment of Nutrition Support Therapy in the Adult Critically Ill Patient: Society of Critical Care Medicine (SCCM) and American Society for Parenteral and Enteral Nutrition (A.S.P.E.N.). JPEN J Parenter Enteral Nutr 40(2): 159211.

7. Singer P, Blaser AR, Berger MM, Alhazzani W, Calder PC, et al. (2019) ESPEN guideline on clinical nutrition in the intensive care unit. Clin Nutr 38(1): 48-79.

8. Singer P, Anbar R, Cohen J, Shapiro H, Shalita Chesner M, et al. (2011). The tight calorie control study (TICACOS): a prospective, randomized, controlled pilot study of nutritional support in critically ill patients. Intensive Care Med 37(4): 601-619.

9. Arabi Y, Jawdat D, Bouchama A, Tamim H, Tamimi W, et al. (2019) Permissive underfeeding, cytokine profiles and outcomes in critically ill patients. PLoS One 14(1): e0209669.

10. Preiser JC, Van Zanten AR, Berger MM, Biolo G, Casaer MP, et al. (2015) Metabolic and nutritional support of critically ill patients: consensus and controversies. Crit Care 19(1): 35.

11. Jung YT, Park JY, Jeon J, Kim MJ, Lee JG (2018) Association of inadequate calorie supplementation with 30-day mortality in critically ill postoperative patients with high modified NUTRIC score. Nutrients 10 (11): 1589.

12. Mulherin DW, Cogle SV. (2017) Updates in Nutrition Support for Critically Ill Adult Patients. Hosp Pharm 52(1): 17-26. 
13. Pontzer H, Durazo Arvizu R, Dugas LR, Plange Rhule J, Bovet P, et al. (2016) Constrained Total Energy Expenditure and Metabolic Adaptation to Physical Activity in Adult Humans. Curr Biol 26(3): 410-417.

14. Zusman O, Thella M, Cohen J, Kagan I, Bendavid I, et al. (2016) Resting energy expenditure, calorie and protein consumption in critically ill patients: a retrospective cohort study. Crit Care 20 (1): 367.

15. Stuever DK, Kidner RF, Chae FE, Evans DC. (2018) Full nutrition or not? Nutr Clin Prac 33 (3): 333-338.

16. Perman MI, Ciapponi A, Franco JV, Loudet C, Crivelli A, et al. (2018) Prescribed hypocaloric nutrition support for critically ill adults. Cochrane Database Syst Rev 6: CD007867.

\section{ISSN: 2574-1241}

DOI: 10.26717/BJSTR.2019.22.003716

Teruyoshi Amagai. Biomed J Sci \& Tech Res

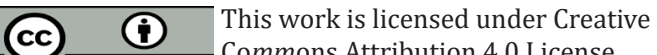

Submission Link: https://biomedres.us/submit-manuscript.php
17. Liu Y, Zhao W, Chen W, Shen X, Fu R, et al. (2018) Effects of early enteral nutrition on immune function and prognosis of patients with Sepsis on mechanical ventilation. J Intensive Care Med 1: 885066618809893.

18. Elke G, Van Zanten AR, Lemieux M, Mc Call M, Jeejeebhoy KN, et al. (2016) Enteral versus parenteral nutrition in critically ill patients: an updated systematic review and meta-analysis of randomized controlled trials. Crit Care 20(1): 117.

19. Estaki M, De Coffe D, Gibson DL. (2014) Interplay between intestinal alkaline phosphatase, diet, gut microbes and immunity. World J Gastroenterol 20(42): 15650-15656.

20. Lallès JP. (2014) Intestinal alkaline phosphatase: novel functions and protective effects. Nutr Rev 72(2): 82-94.

Assets of Publishing with us
RESEARCHES $\quad \begin{aligned} & \text { Global archiving of articles } \\ & \text { - Immediate, unrestricted online access }\end{aligned}$

